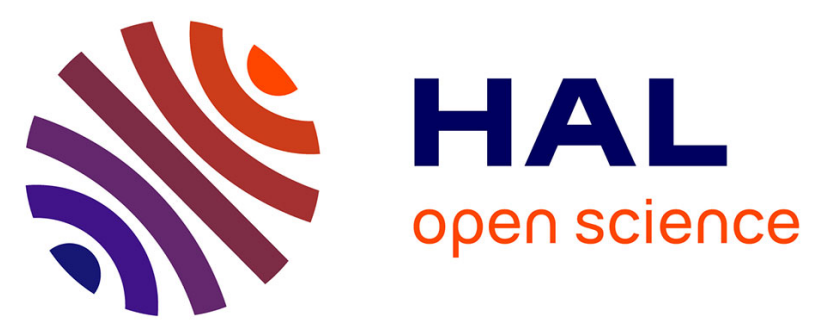

\title{
Polynomial Chaos Expansion for Global Sensitivity Analysis applied to a model of radionuclide migration in randomly heterogeneous aquifers
}

V. Ciriello, V. Di Federico, M. Riva, F. Cadini, J. de Sanctis, Enrico Zio, Alberto Guadagnini

\section{To cite this version:}

V. Ciriello, V. Di Federico, M. Riva, F. Cadini, J. de Sanctis, et al.. Polynomial Chaos Expansion for Global Sensitivity Analysis applied to a model of radionuclide migration in randomly heterogeneous aquifers. Stochastic Environmental Research and Risk Assessment, 2013, 27 (4), pp.945-954. 10.1007/s00477-012-0616-7 . hal-00926341

\section{HAL Id: hal-00926341}

https://hal-centralesupelec.archives-ouvertes.fr/hal-00926341

Submitted on 9 Jan 2014

HAL is a multi-disciplinary open access archive for the deposit and dissemination of scientific research documents, whether they are published or not. The documents may come from teaching and research institutions in France or abroad, or from public or private research centers.
L'archive ouverte pluridisciplinaire HAL, est destinée au dépôt et à la diffusion de documents scientifiques de niveau recherche, publiés ou non, émanant des établissements d'enseignement et de recherche français ou étrangers, des laboratoires publics ou privés. 


\section{Polynomial Chaos Expansion for Global}

2 Sensitivity Analysis applied to a model of

3 radionuclide migration in randomly

4 heterogeneous aquifers

Valentina Ciriello ${ }^{\text {a, }}$, Vittorio Di Federico ${ }^{a}$, Monica Riva ${ }^{b}$, Francesco Cadini ${ }^{c}$,

7 Jacopo De Sanctis ${ }^{\mathrm{c}}$, Enrico Zio ${ }^{\mathrm{c}, \mathrm{d}}$, and Alberto Guadagnini ${ }^{\mathrm{b}}$

${ }^{a}$ Dipartimento di Ingegneria Civile, Ambientale e dei Materiali, Università di

10 Bologna, Italy.

$11{ }^{b}$ Dipartimento di Ingegneria Idraulica, Ambientale, Infrastrutture Viarie,

12 Rilevamento, Politecnico di Milano, Milano, Italy.

$13{ }^{c}$ Dipartimento di Energia, Politecnico di Milano, Milano, Italy.

$14{ }^{d}$ Chair on Systems Science and the Energetic challenge,

15 European Foundation for New Energy-Électricité de France

16 Ecole Centrale Paris and Supelec.

18 *Corresponding author: Tel.: +39 051 2093753; Fax: +39 0512093263.

19 E-mail: valentina.ciriello3@unibo.it

Abstract

We perform Global Sensitivity Analysis (GSA) through Polynomial Chaos Expansion (PCE) on a contaminant transport model for the assessment of radionuclide concentration at a given control location in a heterogeneous aquifer, following a release from a near surface repository of radioactive waste. The aquifer hydraulic conductivity is modeled as a stationary stochastic process in space. We examine the uncertainty in the first two (ensemble) moments of the peak concentration, as a consequence of incomplete knowledge of (a) the parameters characterizing the variogram of hydraulic conductivity, (b) the partition coefficient associated with the migrating radionuclide, (c) the effective dispersivity at the scale of interest. These quantities are treated as random variables and a variance-based GSA is performed in a numerical Monte Carlo framework. This entails solving groundwater flow and transport processes within an ensemble of hydraulic conductivity realizations generated upon sampling the space of the considered random variables. The Sobol indices are adopted as sensitivity measures to provide an estimate of the role of uncertain parameters on the (ensemble) target moments of the variable of interest. The calculation of the indices is performed by employing PCE as a surrogate model of the migration process to reduce the computational burden. We show that the proposed methodology (a) allows identifying the influence of uncertain parameters on key statistical moments of the peak concentration (b) enables extending the number of Monte Carlo iterations to attain convergence of the (ensemble) target moments and (c) leads to considerable saving of computational time while keeping acceptable accuracy. 


\section{Introduction}

46 Performance assessment of radioactive waste repositories aims at evaluating the risk 47 of groundwater contamination due to potential release of radionuclides. Modeling the 48 whole chain of processes involved in this analysis is extremely challenging and requires 49 employing highly complex theoretical and numerical models to couple radionuclide 50 migration within the repository and in the groundwater environment. Uncertainty 51 associated with, e.g., incomplete knowledge of initial and boundary conditions, nature 52 and structure of the groundwater system and related key parameters must be added to the 53 list of difficulties (e.g., Tartakovsky 2007; Winter 2010; Volkova et al. 2008 and 54 references therein).

55 We consider the analysis of the uncertainty associated with the first two (statistical) 56 moments of the peak solute concentration detected at a given location and time. The 57 source of uncertainty is incomplete/imprecise knowledge of the values of the 58 hydrogeological parameters characterizing the system (Rubin 2003; Zhang 2002). For a 59 rational management of the uncertainty analysis, we use Global Sensitivity Analysis 60 (GSA) to obtain information on the relative effects of the uncertain input parameters on 61 the model outputs (Saltelli et al. 2000). In particular, we resort to variance-based 62 methods, which can provide a comprehensive view on the uncertainty and allow 63 identifying the relative and joint contributions of the uncertain input parameters to the 64 uncertainty (variance) of the model outputs (Archer et al. 1997).

65 Within variance-based GSA, the Sobol indices are widely used as sensitivity metrics 66 (Sobol 1993), because they do not require any assumption of linearity in the interpretive 67 model adopted. Their estimation is traditionally performed by Monte Carlo (MC) 68 sampling (Sobol 2001). The sample size needed to attain statistical convergence of the 69 Monte Carlo estimates can be rather large, depending on the complexity and dimension 70 (number of uncertain input parameters) of the problem (e.g., Ballio and Guadagnini 2004, 71 Zhang et al. 2010, and references therein). This might result in a serious and sometimes 72 unsustainable computational burden in cases where repeated high-resolution simulations 73 of the model are required (Sudret 2008).

74 Techniques based on advanced sampling strategies can be introduced to reduce the 75 computational cost associated with Monte Carlo simulations. Among these, the Stochastic 76 Finite Element Method (SFEM) (Ghanem and Spanos 1991) is based on a spectral 77 analysis that allows the expansion of the model output into the probabilistic space, called 78 Polynomial Chaos (PC) (Wiener 1938). The Polynomial Chaos Expansion (PCE) of the 79 model can be used to build a surrogate model such that the variability of the output is 80 represented in the ensemble of the expansion coefficients (Sudret 2008). Once the 
81 surrogate model has been derived, the calculation of the Sobol indices does not add

82 significant extra computational costs. The formulation of a surrogate model in a

83 polynomial form has the additional advantage of allowing performing Monte Carlo

84 simulations with negligible computational effort, as compared to the original, high-

85 complexity model.

86 In this work, we rely on PCE to analyze the uncertainty affecting the outputs of a 87 numerical model of radionuclide migration in an aquifer, following a release from a near 88 surface repository. The outflow from the repository is modeled within the Monte Carlo

89 framework proposed by Cadini et al. (2012). Radionuclide migration in the aquifer is 90 modeled through an Advection-Dispersion-Reaction-Equation (ADRE). The aquifer

91 hydraulic conductivity constitutes a (second-order stationary) randomly heterogeneous

92 field. In this context, the model outputs of interest are the first two (statistical) moments

93 (i.e., mean and variance) of the peak concentration at a given control location in the

94 aquifer. We study how the incomplete/imprecise knowledge of (a) the correlation scale,

$95 \lambda$, of the variogram of the log-conductivity field, (b) the partition coefficient associated

96 with the migrating radionuclide, $k_{d}$, and (c) the effective longitudinal dispersivity at the

97 scale of interest, $\alpha_{L}$, propagates to the selected (ensemble) moments of the output 98 distribution.

99 GSA is performed jointly with PCE to compute the Sobol indices associated with the 100 three uncertain parameters $\left(\lambda, k_{d}, \alpha_{L}\right)$, which are treated as random variables. The PCE 101 based surrogate model is then employed to perform an exhaustive set of Monte Carlo 102 (MC) simulations to attain convergence for the target moments of interest. Given the 103 prohibitive computational costs involved in performing a large number of MC 104 simulations on the original flow and transport model, the goodness of PCE-based results 105 is then assessed on the basis of a limited number of simulations, obtained upon sampling 106 the selected random parameter space.

\section{Theoretical Background and Methodology}

\subsection{Variance-based approaches for GSA}

In this context, the ANOVA (ANalysis Of VAriance) representation of a model

110 output (Archer et al. 1997) is a useful tool for the definition of the Sobol indices (Sobol 111 1993; Archer et al. 1997).

112 Consider a model function $y=f(\mathbf{x}), y$ being a target random response of the 113 model at a prescribed space-time location. This response depends on the vector $\mathbf{x}$ of $n$ 114 independent random model parameters defined in the $n$-dimensional unit hypercube, $I^{n}$.

115 If $f(\mathbf{x})$ is integrable, the following representation holds: 


$$
f(\mathbf{x})=f_{0}+\sum_{i} f_{i}\left(x_{i}\right)+\sum_{i<j} f_{i j}\left(x_{i}, x_{j}\right)+\ldots+f_{1,2, \ldots n}\left(x_{1}, x_{2}, \ldots x_{n}\right)
$$

$$
\int_{0}^{1} f_{i_{1}, \ldots i_{s}}\left(x_{i_{1}}, \ldots x_{i_{s}}\right) d x_{k}=0, \quad k=i_{1}, \ldots i_{s}
$$

118 where $1 \leq i_{1}<\ldots<i_{s} \leq n(s=1, \ldots, n)$ are the indices specifying the parameters upon which

119 each term depends and the $2^{n}$ summands in (1) are orthogonal functions that can be 120 expressed as integrals of $f(\mathbf{x})$, e.g. $f_{0}=\int f(\mathbf{x}) d \mathbf{x}$ is the mean of the model,

$121 f_{i}\left(x_{i}\right)=\int f(\mathbf{x}) \prod_{k \neq i} d \mathbf{x}_{k}-f_{0}$ and so on. Therefore condition (2) renders representation (1),

122 which is typically termed ANOVA decomposition, unique.

123 When $f(\mathbf{x})$ belongs to the space of square-integrable functions, then the total

124 variance, $V$, of the model due to the uncertainty of its parameters is:

$$
V=\int f^{2}(\mathbf{x}) d \mathbf{x}-f_{0}^{2}=\sum_{s=1}^{n} \sum_{i_{1}<\ldots<i_{s}}^{n} V_{i_{1}, \ldots i_{s}}, \quad V_{i_{1}, \ldots i_{s}}=\int f_{i_{1}, \ldots i_{s}}^{2} d x_{i_{1}} \ldots d x_{i_{s}}
$$

$126 V_{i_{1}, \ldots i_{s}}$ being the partial variance, expressing the contribution to $V$ due to the interaction of

127 the set of model parameters $\left\{x_{i_{1}}, \ldots x_{i_{s}}\right\}$. The generic $s$-order Sobol index $S_{i_{1}, \ldots i_{s}}$ is defined 128 as (Sobol 1993):

$$
S_{i_{1}, \ldots i_{s}}=V_{i_{1}, \ldots i_{s}} / V
$$

The sum of the indices defined in (4) is unity. The first-order or principal sensitivity indices, $S_{i}$, describe the significance of each parameter individually considered. Higher-order indices describe the effects of interactions among parameters.

133 The overall effect of a given parameter $x_{i}$ is described by the total sensitivity index $S_{T_{i}}$, 134 defined as:

$$
S_{T_{i}}=\sum_{\eta_{i}} S_{i_{1}, \ldots i_{s}}, \quad \eta_{i}=\left\{\left(i_{1}, \ldots i_{s}\right): \exists k, 1 \leq k \leq s, i_{k}=i\right\} .
$$

A complete GSA requires the estimation of $2^{n}$ integrals of the kind in (3). This is 137 usually done by Monte Carlo simulation (Sobol 2001), but the computational cost 138 becomes prohibitive when the model is complex and the number of uncertain parameters 139 is large (Sudret 2008).

\subsection{Polynomial Chaos Expansion representation of a stochastic model}

142 We focus on the identification of a surrogate model (or metamodel) of a high 143 complexity model (which is hereafter termed full system model) by the Polynomial 144 Chaos Expansion (PCE) technique. This involves the projection of the model equation 
145 into a probabilistic space, termed Polynomial Chaos, to construct an approximation of the 146 model response surface. Wiener (1938) showed that the expansion performed by adopting 147 Hermite Polynomials as a basis converges, in $L_{2}$-sense, for any random process 148 characterized by finite second-order moments. While the Hermite basis is suitable for 149 Gaussian processes, different types of orthogonal polynomials are required for optimum 150 convergence rate in the case of non-Gaussian processes (Xiu and Karniadakis 2002).

151 In this framework, one starts by noting that any square-integrable random model 152 response, $S$, admits the following expansion, or chaos representation (Soize and Ghanem 153 2004):

$$
S=\sum_{j=0}^{\infty} s_{j} \Psi_{j}\left(\left\{\zeta_{n}\right\}_{n=1}^{\infty}\right)
$$

155 Here, $\Psi_{j}$ denotes the $j$-order multivariate orthogonal polynomial, $\left\{\zeta_{n}\right\}_{n=1}^{\infty}$ is the set of 156 independent random variables whose distribution is linked to the choice of the 157 polynomial basis (Xiu and Karniadakis, 2002), and $s_{j}$ are the polynomial coefficients.

In various engineering fields one typically considers stochastic models associated 159 with a finite number $M$ of input random variables. The PCE of the random model output 160 can be derived by approximating (6) to polynomials of degree not exceeding $p$ as

$$
S\left(x_{1}, \ldots x_{M}\right) \cong \sum_{j=0}^{P-1} s_{j} \Psi_{j}\left(\zeta_{1}, \ldots \zeta_{M}\right), \quad P=\frac{(M+p) !}{M ! p !}
$$
where $P$ is the number of (unknown) polynomial coefficients.

The distribution of the input random variables of the model, included in vector $\mathbf{x}$, 164 does not affect the applicability of the method. Note that in cases where this distribution 165 is not interpreted by the one required by the chosen polynomial basis, an isoprobabilistic 166 transformation is required to relate $\mathbf{x}$ and $\zeta=\left(\zeta_{1}, \ldots, \zeta_{M}\right)$. Correlation amongst random 167 input model parameters can be accommodated in the methodology by applying the Nataf 168 transformation (Nataf 1962), for which the knowledge of the marginal probability density 169 functions of the parameters and the associated correlation matrix is required.

170 Assessment of the coefficients $s_{j}$ in (7) can be performed by regression, upon 171 minimization of the variance of a residual defined as the difference between the surrogate

172 model response, $\tilde{S}$, and the exact solution given by the original model (Sudret 2008)

$$
\varepsilon=S(\mathbf{x})-\tilde{S}(\zeta)=S(\mathbf{x})-\sum_{j=0}^{P-1} s_{j} \Psi_{j}(\zeta)
$$

174 Minimization with respect to the vector of the unknown coefficients $\varsigma$ renders 


$$
\boldsymbol{s}=\operatorname{Min}\left\{E\left[(S(\mathbf{x})-\tilde{S}(\zeta))^{2}\right]\right\}
$$

176 with $E[\cdot]$ denoting expected value. It is useful to rewrite (9) as

$$
\boldsymbol{\varsigma}=\left(\boldsymbol{\Psi}^{T} \boldsymbol{\Psi}\right)^{-1} \boldsymbol{\Psi}^{T} \mathbf{S}^{\prime}, \quad \boldsymbol{\Psi}_{i j}=\Psi_{j}\left(\zeta^{i}\right), \quad i=1, \ldots N ; j=0, \ldots P-1
$$

178 where $N$ is the number of regression points, $\mathbf{S}^{\prime}$ is the vector denoting the model response

179 at these points, while the product $\boldsymbol{\Psi}^{T} \boldsymbol{\Psi}$ defines the so-called information matrix.

180 The choice of the optimum set of regression points is performed following the 181 same criterion adopted in the context of integral estimation by Gaussian quadrature 182 (Huang et al. 2007). Solving (10) requires a minimum of $N=P$ regression points. One 183 typically selects $N>P$ to avoid singularity in the information matrix.

\subsection{Polynomial Chaos Expansion and Global Sensitivity Analysis}

Polynomial Chaos Expansion can be considered as a powerful tool for Global Sensitivity Analysis because the entire variability of the original model is conserved in the set of PCE coefficients (Ghanem and Spanos 1991). The Sobol indices can be analytically calculated from these coefficients without additional computational cost (Sudret 2008). Manipulating $\tilde{S}$ by appropriate grouping of terms allows isolating the

$$
\begin{aligned}
& \tilde{S}(\zeta)=s_{0}+\sum_{i=1}^{n} \sum_{\alpha \in \varphi_{i}} s_{\alpha} \Psi_{\alpha}\left(\zeta_{i}\right)+\sum_{1 \leq i_{1}<\ldots<i_{s} \leq n \alpha \in \varphi_{i_{1}, i_{s}}}^{n} s_{\alpha} \Psi_{\alpha}\left(\zeta_{i_{1}}, \ldots, \zeta_{i_{s}}\right)+\ldots \\
& +\sum_{\alpha \in \varphi_{1,2, \ldots n}} s_{\alpha} \Psi_{\alpha}\left(\zeta_{1}, \ldots, \zeta_{n}\right)
\end{aligned}
$$

192 where $\varphi$ denotes a general term depending only on the variables specified by the 193 subscript.

194 In this sense, a PCE is similar to the ANOVA representation of the model. 195 Orthogonality of the polynomial basis allows recognizing that the mean of the model 196 response coincides with the coefficient of the zero-order term, $s_{0}$, in (11) while the total 197 variance of the response is

$$
V_{\tilde{S}}=\operatorname{Var}\left[\sum_{j=0}^{P-1} s_{j} \Psi_{j}(\zeta)\right]=\sum_{j=1}^{P-1} s_{j}^{2} E\left[\Psi_{j}^{2}(\zeta)\right]
$$

$$
S_{i_{1}, \ldots i_{s}}=\frac{\sum_{\alpha \in \varphi_{i_{1} . . i_{s}}} s_{\alpha}^{2} E\left[\Psi_{\alpha}^{2}\right]}{V_{\tilde{S}}}
$$

201 calculation of $E\left[\Psi_{\alpha}^{2}\right\rfloor$ can be performed, e.g., according to Abramowitz and Stegun (1970). 


\section{Application to a model of radionuclide migration in a randomly heterogeneous aquifer}

We exemplify our approach by considering an environmental problem related to the performance assessment of a radioactive waste repository. We use a Monte Carlo simulation model to describe radionuclide release at the repository scale. This model of release of radionuclides, i.e., ${ }^{239} \mathrm{Pu}$, from the repository is linked to a groundwater flow and transport numerical model to simulate radionuclide migration within a heterogeneous aquifer.

The aquifer hydraulic conductivity is modeled as a second-order stationary stochastic process in space. We take the first two (statistical) moments (i.e., mean and variance) of the peak concentration detected at a given control location in the aquifer, as

213 the target model responses. Uncertainty in these variables is considered to be a

214 consequence of incomplete knowledge of (a) the correlation scale of the variogram of the

$215 \log$-conductivity field (b) the partition coefficient associated with the migrating

216 radionuclide, and (c) the effective dispersivity at the scale of interest.

\subsection{Repository representation and modeling of radionuclide release history}

The conceptual repository design considered in the performance assessment illustrated in this study has been proposed by ENEA (Marseguerra et al. 2001a, b) and has similarities with the currently operative disposal facility of El Cabril in Spain (Zuolaga 222 2006).

223 We model the repository as a one-dimensional (along the vertical direction) system (Cadini et al. 2012). The major containment structures of the disposal facility are the waste packages, the modules or containers, the cells and the disposal units. These constitute a multiple-barrier system designed to limit water infiltration and subsequent radionuclide migration. Figure 1a depicts a typical waste package consisting in a steel

228 drum containing the radioactive waste and immobilized in a concrete matrix. The

229 diameter and the height of the waste package have been set respectively to $0.791 \mathrm{~m}$ and

$2301.1 \mathrm{~m}$, for a total volumetric capacity of around 4001 . Figure $1 \mathrm{~b}$ shows a cross-section of 231 the containment module adopted in this study, i.e., a concrete box-shaped structure which 232 contains 6 waste packages and is sealed with a concrete top cover. The empty spaces 233 between the packages are filled by bentonite. The external length of the module is $3.05 \mathrm{~m}$, 234 with a width and height of $2.09 \mathrm{~m}$ and $1.7 \mathrm{~m}$, respectively. The corresponding internal 235 dimensions are $2.75 \mathrm{~m}, 1.79 \mathrm{~m}$ and $1.37 \mathrm{~m}$. The modules are arranged in $5 \times 6 \times 8$ arrays within concrete structure cells built below the natural ground level. 
Figure 2 depicts the modules arrangement and the typical repository placement at

238 a given site. The disposal unit is a concrete structure embedding a row of 6 to 10 cells.

239 The disposal facility comprises several units, which are typically arranged into parallel

240 rows. Each unit can be modeled as an independent system which can be built and

241 operated without interfering with the remaining units.

242 In agreement with typical engineering scenarios we consider that (Marseguerra et

243 al. 2001a, b): (i) the modules are identical; (ii) the mass transport occurs chiefly along the

244 vertical direction; and (iii) lateral diffusive spreading is symmetric. Under these

245 assumptions, estimating the probability of radionuclide release into the groundwater

246 system below the repository can be reduced to the one-dimensional problem of estimating

247 the release from a column of five identical vertically stacked modules, i.e., the repository

248 column may be envisioned as a one-dimensional array of compartments, each

249 corresponding to a module.

250 The radionuclides transition across the compartments is described stochastically.

251 Under the assumption that solute displacement can be modeled as a Markovian process,

252 the transition rates can be identified from the classical advection/dispersion equation.

253 Non-Fickian transport can be modeled according to existing conceptual schemes

254 (Berkowitz et al. 2006 and references therein) where the relevant transport parameters

255 could be estimated by detailed data analysis at the temporal and spatial scales at which

256 the processes of interest occur.

257 For the purpose of our example we adopt the following criteria, which can be

258 considered as conservative in a performance assessment protocol: (i) the protection

259 offered by the concrete cell roof and ceiling and the backfill layers fails; (ii) the whole

260 column, which is formed by 5 modules, is saturated and a constant water head of $0.15 \mathrm{~m}$

261 is applied at the top of the highest module, i.e., the water head at the top of the column is

$262 h(z=5 \times 1.7 \mathrm{~m})=8.65 \mathrm{~m}$; (iii) the water head at the bottom of the column is zero; (iv)

263 each module is subject to constant head gradient $\Delta h / \Delta z=1.018$, where $\Delta h=8.65 \mathrm{~m}$ and

$264 \Delta z=5 \times 1.7 \mathrm{~m}=8.5 \mathrm{~m}$ is the column height; (v) the ${ }^{239} \mathrm{Pu}$ radioactive decay and the

265 subsequent generation of other radionuclides from the decay chains are neglected within

266 the repository; (vi) the migration of ${ }^{239} \mathrm{Pu}$ occurs at linear isothermal equilibrium.

267 The numerical code MASCOT (Marseguerra and Zio 2001; Marseguerra et al.

268 2003; Cadini et al. 2012) has been adopted to compute the probability density function of

269 the release of ${ }^{239} \mathrm{Pu}$ from the modules. Details of the computations and the resulting

270 temporal dynamics of the radionuclide release history are presented in Cadini et al.

271 (2012). 


\section{$272 \quad 3.2 \quad$ Radionuclide migration in the groundwater system}

For simplicity and for the purpose of our illustration we disregard the

274 radionuclide transfer time within the partially saturated zone and analyze only

275 contaminant residence time within the fully saturated medium. This assumption may be

276 regarded as conservative because it leads to overestimating the radionuclide concentration

277 detected downstream of the repository. This can also be considered as a viable working

278 assumption in the presence of shallow reservoirs. The effect of processes occurring within

279 the partially saturated region may require an additional analysis, which is outside the

280 scope of this work.

281 Groundwater flow and contaminant transport are modeled within a two282 dimensional system. The (natural) log-transformed hydraulic conductivity, $Y(\boldsymbol{x})(\boldsymbol{x}$ 283 denoting the space coordinates vector), is modeled as a second-order stationary spatial 284 random function. For our example, the parameters of the variogram of $Y$ have been 285 selected as representative of a field case study, which we do not specifically report for 286 confidentiality reasons. We note, however, that the particular choice of these values does 287 not affect the generality of the methodology. Log-conductivity is characterized by an 288 isotropic variogram of the exponential type, with sill $\sigma^{2}=1.21$. For the purpose of our 289 illustrative example, we set the variogram sill and consider its correlation scale as an 290 uncertain parameter (see Section 4) because of its poor identifiability due to typical 291 horizontal spacing of available field-scale measuring locations. Monte Carlo realizations 292 of $Y(\boldsymbol{x})$ have been performed by employing the sequential Gaussian scheme implemented 293 in the code GCOSIM3D (Gómez-Hernández and Journel 1993).

294 We consider a two-dimensional domain of uniform lateral side equal to $2000 \mathrm{~m}$.

295 As an example, a selected realization of the log-conductivity distribution is depicted in 296 Figure 3 together with the repository projection (R), with sides equal to $50 \mathrm{~m}$ and $80 \mathrm{~m}$, 297 and the target control point (W), located $960 \mathrm{~m}$ downstream of the repository fence line.

298 The domain is discretized into square cells with uniform side of $10 \mathrm{~m}$, ensuring 299 that there are at least five log-conductivity generation points per correlation scale (see 300 Section 4 for additional details). Each of the $8 \times 5$ cells located under the repository 301 projection area receives the release of a cluster of $4 \times 3$ columns of 5 modules. These 302 cells are modeled through a recharge boundary condition so that a time-dependent influx 303 solute mass is injected in the porous medium according to a suitable discretization in time 304 of the Monte Carlo-based outflow from the repository. As in Cadini et al. (2012), we set 305 the incoming water flow $\left[\mathrm{m}^{3} / \mathrm{y}\right]$ from the repository at a constant value equal to $306 \Phi_{i n}=q_{d} S, \quad q_{d}=21.2[\mathrm{~m} / \mathrm{y}]$ being the water Darcy flux at the bottom of the 5 modules 307 column and $S\left[\mathrm{~m}^{2}\right]$ being the area of the source cells. The associated radionuclide 308 concentration $\left[\mathrm{Bq} / \mathrm{m}^{3}\right]$ released to the aquifer is then: 
310 where $A_{0}=1.6 \times 10^{6}[\mathrm{~Bq}]$ is the total activity of ${ }^{239} \mathrm{Pu}$ (which we assumed to be

311 uniformly distributed) in the repository at a reference time $t=0$ and $p d f_{\text {out }}(t)\left[\mathrm{y}^{-1}\right]$ is the 312 release probability density function from the four compartment domain (i.e., the five 313 module column). The adopted ${ }^{239} \mathrm{Pu}$ activity level corresponds to the Italian inventory 314 (Enea 2000) and justifies the assumption of disregarding solubility-limited release. In our 315 example, the concentration of ${ }^{239} \mathrm{Pu}$ within the repository is $C_{\text {rep }}^{\text {Pu239 }} \cong \frac{\lambda_{r} A_{0}}{N_{A} V_{\text {rep }}}=2.96 \cdot 10^{-14}<C_{\text {sl }}^{\text {Pu239 }}=2.30 \cdot 10^{-4}\left[\mathrm{~mol} / \mathrm{m}^{3}\right]$, where $\lambda_{r}=0.28761 \cdot 10^{-4}\left[\mathrm{y}^{-1}\right]$ is

317 the ${ }^{239} \mathrm{Pu}$ constant decay, $N_{A}$ is the Avogadro constant, $V_{\text {rep }}$ is the total volume of the 318 repository and $C_{s l}^{P u 239}$ is the solubility limit of ${ }^{239} \mathrm{Pu}$. Additional details are presented in 319 Cadini et al. (2012).

320 Base groundwater flow in the aquifer is driven by a constant hydraulic head drop 321 between the East and West boundaries, resulting in a unit average head gradient. No-flow 322 conditions are assigned to the North and South boundaries.

Simulations of the steady state flow problem for each conductivity realization are performed with the widely used and thoroughly tested finite difference code MODFLOW2000 (McDonald and Harbaugh 1988). Radionuclide migration in the groundwater system is then modeled by means of the classical Advection-Dispersion

327 Equation (ADE), where the partition coefficient, $k_{d}$, governing sorption of the 328 contaminant onto the host solid matrix and the effective longitudinal dispersivity, $\alpha_{L}$ (for 329 simplicity, transverse dispersivity is assumed to be equal to $0.1 \alpha_{L}$ ), are considered to be 330 random variables, as described in Section 4. A uniform effective porosity of 0.15 is considered.

\section{Global Sensitivity Analysis of the (ensemble) moments of radionuclide peak concentration}

The three random parameters selected for our demonstration are assumed to be uniformly distributed within the intervals reported in Table 1 . The ranges of variability of $\lambda$ and $\alpha_{L}$ are compatible with the selected domain size, and consistent with the lack of a sufficiently large number of closely spaced $Y$ measuring points. The degree of variability of $k_{d}$ has been chosen according to ENEA (1997) and Nair and Krishnamoorthy (1999).

The model response, i.e., the radionuclide peak concentration, $c_{p}$, at the control point is then, in turn, a random variable. As introduced in Section 3, we perform our analysis in a numerical Monte Carlo framework according to the following steps: (a) a set 
342 of $N_{f}=100 Y$ fields are generated by GCOSIM for given values of the random 343 parameters sampled within the intervals presented in Table 1; (b) groundwater flow and

344 transport are solved and (ensemble) mean, $\left\langle c_{p}\right\rangle$, and standard deviation, $\sigma_{c_{p}}$, of the peak 345 concentration are computed; (c) steps (a) and (b) are repeated for different sampling 346 points in the random parameters space; and (d) GSA is performed to discriminate the 347 relative contribution of the random parameters to uncertainty of $\left\langle c_{p}\right\rangle$ and $\sigma_{c_{p}}$. Note that 348 due to the random nature of $Y(\boldsymbol{x})$, we propose to perform GSA on the (ensemble) 349 moments of $c_{p}$ rather than on its actual value calculated at the selected control location 350 for each random realization. Conceptually, this is equivalent to performing a GSA of the 351 results stemming from the solution of transport equations satisfied by the ensemble 352 moments of the evolving concentrations (e.g., Guadagnini and Neuman (2001) and 353 Morales-Casique et al. (2006 a,b) for conservative solutes).

354 The procedure illustrated is rather cumbersome when considering the solution of 355 the full system model, because of the large number of simulations required, so that a GSA 356 might become impractical. Therefore, we adopt the PCE technique presented in Section 2 357 and derive expansions of order $p=2,3$ and 4 , for both $\left\langle c_{p}\right\rangle$ and $\sigma_{c_{p}}$. We resort to the 358 Legendre Chaos space, because the uncertain input parameters are associated with 359 uniform distributions.

360 The calibration of the coefficients of the surrogate models requires $N_{R}=10,38$ 361 and 78 (respectively for $p=2,3,4$ ) sampling points in the space of the three selected 362 uncertain parameters. In our example, this corresponds to $N_{M C}=1000,3800,7800$ runs 363 of the full model of groundwater flow and transport. Calculation of the Sobol indices is 364 then performed with negligible additional computational requirements.

365 Figure 4 reports the Total Sensitivity Indices, $S_{T}$ (left), and variances, $V$ (right), 366 of $\left\langle c_{p}\right\rangle$ versus the degree of polynomial expansion, $p$. Figure 5 reports the corresponding 367 results for $\sigma_{c_{p}}$.

368 We start by noting that $S_{T}$ and $V$ are not dramatically influenced by the degree 369 of polynomial expansion selected for both moments. The good agreement obtained 370 between Total and Principal Sensitivity Indices (not shown) implies that the effects of 371 parameters interactions can be neglected in this example.

$372 \quad$ Figure 4 reveals that $k_{d}$ and $\alpha_{L}$ are the parameters which are most influential to $373\left\langle c_{p}\right\rangle$, regardless of the degree of expansion adopted. On the other hand, the log374 conductivity correlation scale, $\lambda$, and (to a lesser degree) the dispersivity, $\alpha_{L}$, strongly 
375 influence $\sigma_{c_{p}}$, while $k_{d}$ does not have a significant impact for the specific values adopted 376 in the case study. The uncertainty associated with the mean peak concentration is thus 377 related mostly to the spatial structure of heterogeneity and to the strength of the 378 dispersion phenomena, and less to the considered geochemical scenario.

379 The calibrated surrogate models allow extending with negligible computational 380 cost the number of Monte Carlo simulation runs required for computing mean and 381 standard deviation of $\left\langle c_{p}\right\rangle$ and $\sigma_{c_{p}}$, as illustrated in Section 2.2. Figures 6 and 7 382 respectively depict the dependence of the mean and the standard deviation of $\left\langle c_{p}\right\rangle$ and $383 \sigma_{c_{p}}$ on the number of Monte Carlo runs performed with the calibrated surrogate models. 384 The high number $\left(\approx 10^{4}\right)$ of simulations required to attain convergence denotes the 385 complexity of the case study and supports the adoption of a surrogate model to assess the 386 uncertainty associated with the model response at reasonable computational costs.

387 The reliability of the results obtained through the PCE-based surrogate model has 388 been analyzed by comparison against a number of full model runs performed by uniform 389 sampling of $N_{s}=100$ points in the random parameters space, corresponding to a total of $39010^{4}$ random realizations of $Y(\boldsymbol{x})$. The limited amount of sampling points selected is due to 391 the excessive computational cost associated with the full model run (about 4 min for each 392 simulation on a standard computer with a $3.16 \mathrm{GHz}$ processor).

Figure 8 reports the relative fraction, $\mathscr{T}(\%)$, of the mean concentration values,

395 comprised within intervals of width $w= \pm\left(\sigma_{c_{p}}^{F M}\right)_{l}, \pm 2\left(\sigma_{c_{p}}^{F M}\right)_{l}$, and $\pm 3\left(\sigma_{c_{p}}^{F M}\right)_{l}$ centered 396 around $\left\langle c_{p}\right\rangle_{l}^{F M},\left\langle c_{p}\right\rangle_{l}^{F M}$ and $\left(\sigma_{c_{p}}^{F M}\right)_{l}$ respectively being the mean and standard deviation 397 of the peak concentration computed by means of the full system model. The latter is 398 based on a standard Monte Carlo solution of radionuclide migration within $N M C=100$ $399 \log$-conductivity realizations for each $1 \leq l \leq N_{s}$. It can be seen that at least $40 \%$ of the 400 values calculated with the surrogate models of different orders are comprised within the 401 intervals of width $\pm{\sigma_{c_{p}}}^{F M}$, while about $75 \%$ of the results are included within intervals 402 not exceeding $\pm 2{\sigma_{c_{p}}}^{F M}$. According to this criterion, Figure 8 suggests that the best 403 results for our example appear to be provided by the PCE of order $p=2$.

404 To complement these results, Table 2 reports the mean and standard deviation of $405\left\langle c_{p}\right\rangle$ calculated on the basis of the $N_{s}=100$ sampling points in the random parameters 406 space for each model (standard Monte Carlo and surrogate models of different order). 
407 Table 3 reports the corresponding results for $\sigma_{c_{p}}$. The limited number of simulations does 408 not allow to attain convergence of the target moments. However, it is possible to observe 409 that the PCE of order $p=4$ provides the best approximation of both the mean and

410 standard deviation of $\left\langle c_{p}\right\rangle$ calculated with the full model. In other words, the Total

411 Sensitivity Indices for $\left\langle c_{p}\right\rangle$ calculated with the PCE of order $p=4$ are candidates to 412 provide the best indications for a GSA, as one might expect. Finally, it can be noted that 413 the PCE of order $p=3$ best approximates the mean and standard deviation of $\sigma_{c_{p}}$ 414 calculated with the full model on the basis of the simulations performed.

\section{Conclusions}

In this work we proposed an approach for performing a Global Sensitivity

417 Analysis (GSA) of a high-complexity theoretical and numerical model descriptive of the 418 potential release of radionuclides from a near surface radioactive waste repository and 419 their subsequent migration in the groundwater system. We considered uncertainty 420 stemming from incomplete knowledge of the variogram and transport parameters (i.e., the 421 correlation length of the variogram of log-conductivity, the partition coefficient 422 associated with the migrating radionuclide and the effective dispersivity at the scale of 423 interest) and, due to the random nature of the hydraulic conductivity field. We identified 424 as target system responses the first two (ensemble) moments of the peak concentration at 425 a given control point. GSA has been performed through the Polynomial Chaos Expansion 426 (PCE) technique, leading to the following key results: (a) the analysis of the Sobol indices 427 has revealed that the (ensemble) mean of the peak concentration is strongly influenced by 428 the uncertainty in the partition coefficient and the longitudinal dispersivity, and the 429 effects of these parameters shadow the impact of the spatial coherence of the log430 conductivity field at the scale analyzed; (b) on the other hand, the log-conductivity 431 correlation scale is the most influential factor affecting the uncertainty of the standard 432 deviation of the peak concentration in our example; and (c) the PCE surrogate models 433 allow extending, with negligible computational cost and acceptable accuracy, the number 434 of Monte Carlo iterations to attain convergence of the selected target moments.

Our results support the relevance of adopting the proposed model reduction technique for complex numerical models. This methodology allows performing in-depth analyses which would be otherwise unfeasible, thus severely limiting our capability to represent the relevant processes involved in a target environmental scenario.

\section{Acknowledgments}


443 V. Ciriello acknowledges partial support from Marco Polo Program 2011 of the University of 444 Bologna. F. Cadini, J. De Sanctis and E. Zio acknowledge the support from Agenzia Nazionale per 445 le Nuove Tecnologie, l’Energia e lo Sviluppo Economico Sostenibile (ENEA).

446 
Abramowitz M, Stegun IA (1970) Handbook of mathematical functions. Dover Publications, New York.

Archer GEB, Saltelli A, and Sobol IM (1997) Sensitivity measures, ANOVA like techniques and the use of bootstrap. J Stat Comput Simulation 58:99-120.

Ballio F, Guadagnini A (2004) Convergence assessment of numerical Monte Carlo simulations in groundwater hydrology. Water Resour Res 40 W04603.

Berkowitz B, Cortis A, Dentz M, Scher H (2006) Modeling non-Fickian transport in geological formations as a continuous time random walk. Rev of Geophys 44 RG2003.

Cadini F, De Sanctis J, Cherubini A, Zio E, Riva M, Guadagnini A (2012) An integrated simulation framework for the performance assessment of radioactive waste repositories. Annals of Nuclear Energy 39:1-8.

ENEA (1997) Internal Report. Chapman, N. A..

ENEA (2000) Inventario nazionale dei rifiuti radioattivi - Task Force per il sito nazionale di deposito dei materiali radioattivi. 3rd Ed (in Italian).

Ghanem RG, Spanos PD (1991) Stochastic finite elements-a spectral approach. Springer, Berlin.

Gómez-Hernández JJ, Journel AG (1993) Joint sequential simulation of multi-Gaussian field. Geostatitics Troia '92, 1:85-94. Ed Soares.

Guadagnini A, Neuman SP (2001) Recursive conditional moment equations for advective transport in randomly heterogeneous velocity fields. Transp Porous Med 42:37-67.

Huang S, Sankaran M, Ramesh R (2007) Collocation-based stochastic finite element analysis for random field problems. Probabilistic Engineering Mechanics 22:194-205.

Marseguerra M, Zio E, Patelli E, Giacobbo F, Ventura G, Mingrone G (2003) Monte Carlo simulation of contaminant release from a radioactive waste deposit. Math Comput Simul 62:421430 .

Marseguerra M, Patelli E, Zio E (2001) Groundwater contaminant transport in presence of colloids I. A stochastic nonlinear model and parameter identification. Annals of Nuclear Energy 28:777803.

Marseguerra M, Patelli E, Zio E (2001) Groundwater contaminant transport in presence of colloids II. Sensitivity and uncertainty analysis on literature case studies. Annals of Nuclear Energy 28:1799-1807.

Marseguerra M, Zio E (2001) Genetic algorithms for estimating effective parameters in a lumped reactor model for reactivity predictions. Nuclear Science and Engineering 139:96-104.

McDonald MG, Harbaugh AW (1988) A Modular Three-Dimensional Finite-Difference Groundwater Flow Model. Man. 83-875, U.S. Geol. Surv. Reston, VA.

Morales-Casique E, Neuman SP, Guadagnini A (2006) Nonlocal and localized analyses of nonreactive solute transport in bounded randomly heterogeneous porous media: Computational analysis. Adv Water Resour 29:1399-1418.

Morales-Casique E, Neuman SP, Guadagnini A (2006) Nonlocal and localized analyses of nonreactive solute transport in bounded randomly heterogeneous porous media: Theoretical framework. Adv Water Resour 29:1238-1255. 
Nataf A (1962) Détermination des distributions dont les marges sont données. C R Acad Sci $225: 42-3$

Nair RN, Krishnamoorthy TM (1999) Probabilistic safety assessment model for near surface radioactive waste disposal facilities. Environmental Modelling \& Software 14:447-460.

Rubin Y (2003) Applied Stochastic Hydrogeology. Oxford Univ. Press, New York.

Saltelli A, Chan K, Scott EM (2000) Sensitivity analysis. Wiley, New York.

Sobol IM (1993) Sensitivity estimates for nonlinear mathematical models. Math Modeling Comput 1:407-414.

Sobol IM (2001) Global sensitivity indices for nonlinear mathematical models and their Monte Carlo estimates. Math Comput Simulation 55:271-280.

Soize C, Ghanem R (2004) Physical systems with random uncertainties: Chaos representations with arbitrary probability measures. J Sci Comput 26(2):395-410.

Sudret B (2008) Global sensitivity analysis using polynomial chaos expansions. Reliab Eng Syst Safety 93:964-979.

Tartakovsky DM (2007) Probabilistic risk analysis in subsurface hydrology. Geophys Res Lett 34.

Volkova E, Iooss B, Van Dorpe F (2008) Global sensitivity analysis for a numerical model of radionuclide migration from the RRC "Kurchatov Institute" radwaste disposal site. Stoch Environ Res Risk Assess 22:17-31.

Wiener N (1938) The homogeneous chaos. Am J Math 60:897-936.

Winter CL, (2010) Normalized Mahalanobis distance for comparing process-based stochastic models. Stoch Environ Res Risk Assess 24:917-923.

Winter CL, Tartakovsky DM (2002) Groundwater flow in heterogeneous composite aquifers. Water Resour Res 38(8):1148.

Xiu D, Karniadakis GE (2002) The Wiener-Askey polynomial chaos for stochastic differential equations. J Sci Comput 24(2):619-644.

Zhang D, Shi L, Chang H, Yang J (2010) A comparative study of numerical approaches to risk assessment of contaminant transport. Stoch Environ Res Risk Assess 24:971-984.

Zhang D (2002) Stochastic methods for flow in porous media: copying with uncertainties. Academic Press, San Diego.

Zuloaga P (2006) New Developments in LLW Management in Spain. ENRESA.

$\langle$ http://www.euronuclear.org/events/topseal/presentations/PP-Session-IIIZuloaga.pdf>. 
560 Fig. 1 Conceptual design of: (a) a waste package, (b) a containment module (ENEA 1987).

561 Fig. 2 Sketch of the $5 \times 6 \times 8$ array of modules considered in a repository cell (ENEA 1987;

562 Marseguerra et al. 2001a, b).

563 Fig. 3 Sketch of the adopted two-dimensional groundwater flow domain, including the

564 repository projection $(\mathrm{R})$ and the selected control point $(\mathrm{W})$, for a selected realization of the log565 conductivity field.

566 Fig. 4 Total Sensitivity Indices ( $S_{T}(\Omega), \Omega=\lambda, \alpha_{L}, k_{d}$ ), Total Variance ( $V$ ) and Partial

567 Variances ( $V(\Omega), \Omega=\lambda, \alpha_{L}, k_{d}$ ) calculated for $\left\langle c_{p}\right\rangle$ and $p=2,3,4$.

$568 \quad$ Fig. 5 Total Sensitivity Indices ( $S_{T}(\Omega), \Omega=\lambda, \alpha_{L}, k_{d}$ ), Total Variance ( $V$ ) and Partial

569 Variances $\left(V(\Omega), \Omega=\lambda, \alpha_{L}, k_{d}\right.$ ) calculated for $\sigma_{c_{p}}$ and $p=2,3,4$.

570 Fig. 6 Dependence of the (a) mean and (b) standard deviation of $\left\langle c_{p}\right\rangle$ on the number of Monte

571 Carlo iterations performed with the calibrated surrogate models.

572 Fig. 7 Dependence of the (a) mean and (b) standard deviation of $\sigma_{c_{p}}$ on the number of Monte

573 Carlo iterations performed with the calibrated surrogate models.

574 Fig. 8 Relative fraction, $\mathscr{A} \%)$, of the mean concentration values, $\left\langle c_{p}\right\rangle_{l}^{S M}\left(l=1,2, \ldots, N_{s}\right)$

575 calculated with the PCE at different orders $(p=2,3,4)$ which are comprised within intervals of 576 width $w= \pm\left(\sigma_{c_{p}}^{F M}\right)_{l}, \pm 2\left(\sigma_{c_{p}}^{F M}\right)_{l}$, and $\pm 3\left(\sigma_{c_{p}}^{F M}\right)_{l}$ centered around $\left\langle c_{p}\right\rangle_{l}^{F M} ;\left\langle c_{p}\right\rangle_{l}^{F M}$ and

$577 \quad\left(\sigma_{c_{p}}^{F M}\right)_{l}$ respectively are the mean and standard deviation of the peak concentration computed

578 through the full system model on the basis of a standard Monte Carlo analysis of radionuclide 579 migration within $N M C=100 \log$-conductivity realizations for each $l$. 
Table 1 Intervals of variability of the selected uniformly distributed random model parameters.

\begin{tabular}{|l|l|}
\hline \multicolumn{1}{|c|}{ Random Variable } & Distribution \\
\hline Partition Coefficient, $k_{d}$ & $U\left(1 \frac{l}{g} ; 3 \frac{l}{g}\right)$ \\
\hline Longitudinal Dispersivity, $\alpha_{L}$ & $U(50 m ; 70 m)$ \\
\hline Correlation length of log-conductivity, $\lambda$ & $U(40 m ; 100 m)$ \\
\hline
\end{tabular}

584 Table 2 Values of the mean and standard deviation of $\left\langle c_{p}\right\rangle$ calculated with the full model and the

\begin{tabular}{|l|c|c|}
\hline \multicolumn{1}{|c|}{ Model } & Mean of $\left\langle c_{p}\right\rangle$ & Standard Deviation of $\left\langle c_{p}\right\rangle$ \\
\hline Full system model & $2.738 \mathrm{E}-06$ & $3.241 \mathrm{E}-07$ \\
\hline Surrogate model $p=2$ & $2.407 \mathrm{E}-06$ & $7.175 \mathrm{E}-08$ \\
\hline Surrogate model $p=3$ & $3.190 \mathrm{E}-06$ & $1.887 \mathrm{E}-07$ \\
\hline Surrogate model $p=4$ & $2.538 \mathrm{E}-06$ & $3.462 \mathrm{E}-07$ \\
\hline
\end{tabular}

588 Table 3 Values of the mean and standard deviation of $\sigma_{c_{p}}$ calculated with the full system model and the surrogate models on the basis of 100 sampling points in the random parameter space.

\begin{tabular}{|l|c|c|}
\hline \multicolumn{1}{|c|}{ Model } & Mean of $\sigma_{c_{p}}$ & Standard Deviation of $\sigma_{c_{p}}$ \\
\hline Full system model & $4.061 \mathrm{E}-07$ & $8.169 \mathrm{E}-08$ \\
\hline Surrogate model $p=2$ & $4.708 \mathrm{E}-07$ & $3.310 \mathrm{E}-08$ \\
\hline Surrogate model $p=3$ & $4.278 \mathrm{E}-07$ & $5.719 \mathrm{E}-08$ \\
\hline Surrogate model $p=4$ & $4.530 \mathrm{E}-07$ & $1.321 \mathrm{E}-07$ \\
\hline
\end{tabular}

